\title{
Point-of-care ultrasound diagnosis of SCFE
}

\author{
Michael B. Stone $\cdot$ Chameeka Barrett
}

Received: 13 November 2009/Accepted: 14 December 2009/Published online: 8 January 2010

(C) Springer-Verlag 2010

\begin{abstract}
Introduction A 13-year-old boy presented to the emergency department with left knee pain after a fall. Initial radiographs were unremarkable, but the child returned to the emergency department 6 weeks later with persistent symptoms.

Materials and methods A bedside sonogram of the left hip performed by the treating emergency physician demonstrated a widened epiphyseal plate and an associated effusion, consisted with a slipped capital femoral epiphysis (SCFE). Repeat radiographs confirmed the diagnosis.

Conclusion The ultrasound features and test characteristics for the detection of SCFE are reviewed.
\end{abstract}

Keywords Emergency ultrasound - Critical ultrasound . Slipped capital femoral epiphysis - SCFE

\section{Case report}

A 13-year-old male with no prior medical history presented to the emergency department with left knee pain after a mechanical fall from standing. Vital signs were normal and physical examination revealed diffuse tenderness to the left hip and knee. Radiographs of the left knee and pelvis (Fig. 1) were interpreted as unremarkable, and the patient was placed in a knee immobilizer and discharged with orthopedic follow-up.

Approximately 6 weeks later, the patient returned to the emergency room for left hip and knee pain for 14 days and the inability to bear weight. The patient reported having

M. B. Stone $(\bowtie) \cdot$ C. Barrett

Department of Emergency Medicine, SUNY Downstate/Kings

County Hospital Center, Brooklyn, NY 11203, USA

e-mail: drmikestone@gmail.com sustained an additional fall 2 weeks prior to this visit. The patient's temperature was $98.1 \mathrm{~F}$, pulse $82 / \mathrm{min}$, blood pressure $127 / 62 \mathrm{mmHg}$, respirations $16 / \mathrm{min}$ and room air oxygen saturation $100 \%$. The patient was in mild distress due to pain, and the left knee and left hip were diffusely tender to palpation. There was limited active and passive range of motion of the hip, and an antalgic gait. Neurological and vascular exams were normal.

\section{Results and discussion}

Given the patient's previous normal radiographs, the treating emergency physician performed a bedside ultrasound of the left hip using a 5-2 MHz curvilinear transducer (SonoSite MTurbo, Bothell WA) aligned parallel to the femoral neck. A joint effusion was identified and widening of the epiphyseal plate with displacement of the epiphysis from the metaphysis was noted (Fig. 2; asterisk $=$ growth plate, $\mathrm{e}=$ effusion) suggesting a slipped capital femoral epiphysis (SCFE). The contralateral hip was examined for comparison (Fig. 3) and demonstrated a normal contour with no displacement of the epiphysis and no widening of the epiphyseal plate. Radiographs obtained on this visit confirmed the diagnosis of SCFE (Fig. 4). The patient was admitted to the pediatric orthopedic service for operative management.

Emergency physicians must promptly identify patients with a SCFE, as delays in this diagnosis are associated with increased slip severity and morbidity [1]. Unfortunately, plain radiography including an antero-posterior pelvis view and a frog-leg lateral hip view has a poor overall sensitivity, ranging from 40 to $79 \%$ [2]. Ultrasound has been previously described as an alternative to plain radiography for the diagnosis of SCFE [3], with reported sensitivities as 


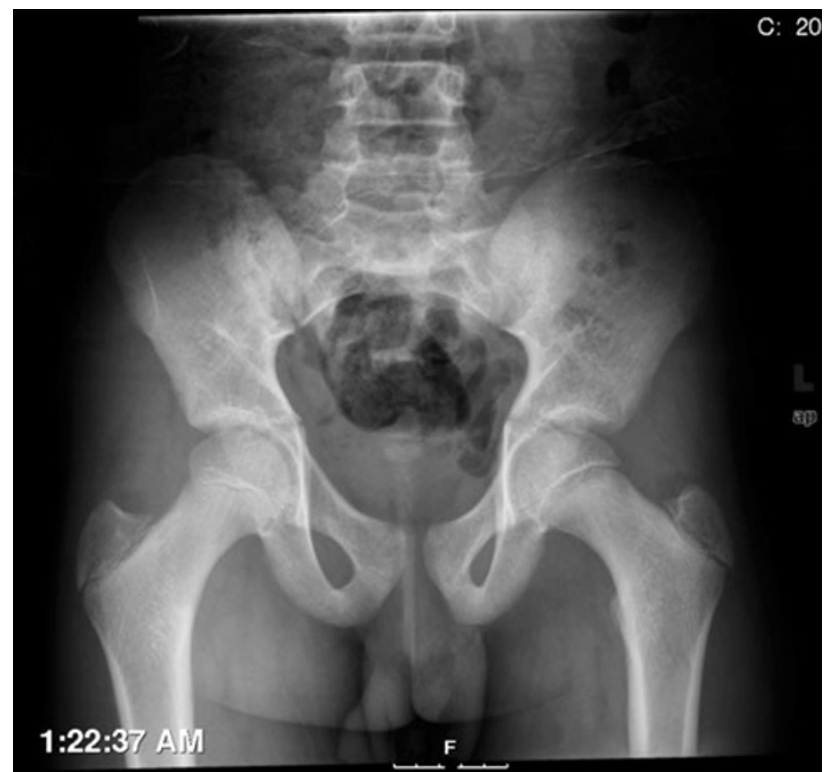

Fig. 1 Antero-posterior radiograph of the pelvis obtained on initial visit

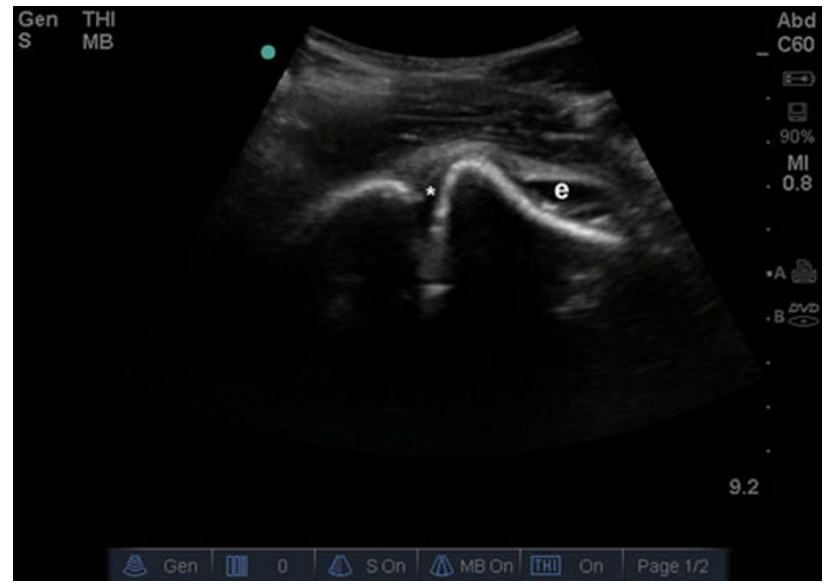

Fig. 2 Bedside ultrasound of the left hip (asterisk growth plate, $e$ effusion)

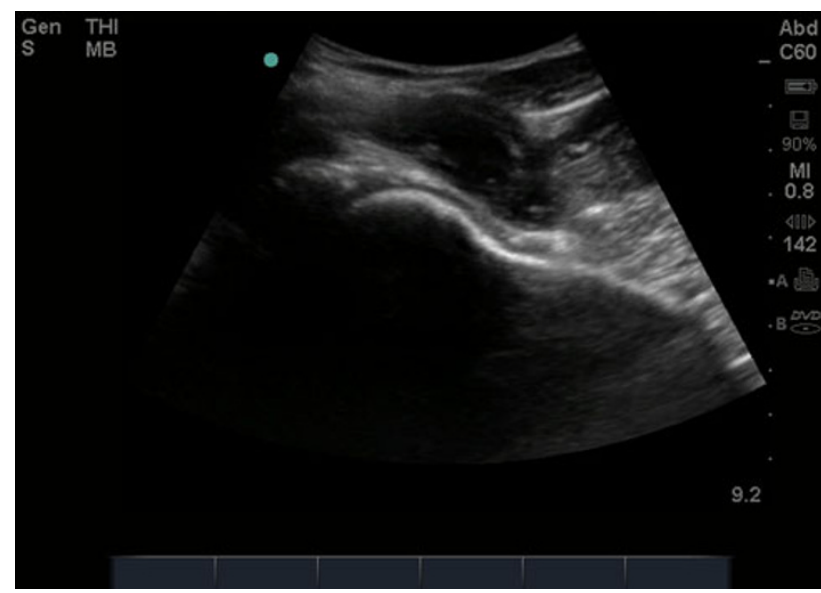

Fig. 3 Bedside ultrasound of the right hip

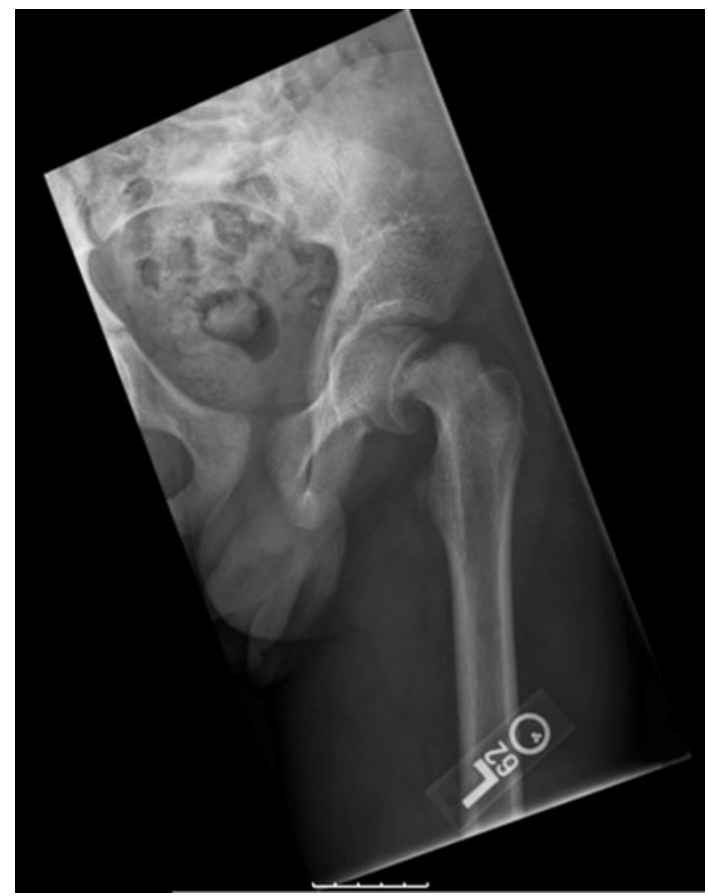

Fig. 4 Antero-posterior radiograph of the left hip

high as $95 \%$ in one study [4]. In addition, when compared to plain radiography ultrasound allows for earlier diagnosis of SCFE, and for more accurate grading of the degree of physeal displacement [4]. Some authors have reported improved sensitivity of ultrasound when compared to plain radiography for not only the detection of SCFE, but also the confirmation of surgical reduction [5].

The ultrasound examination for SCFE may be performed with either a low- or high-frequency transducer; key findings include an associated joint effusion, widening of the growth plate, and posterior displacement of the epiphysis relative to the metaphysis. Our focused assessment during this examination was to determine the presence or absence of SCFE, and therefore in this case, the degree of posterior epiphyseal displacement was not measured. However, it should be noted that the degree of posterior epiphyseal displacement appears to correlate with the severity of the slip when compared to plain radiography [6]. The ability to compare findings to the contralateral side is of significant value to the operator, yet providers must remember that SCFE may occur bilaterally and that symptoms can be vague.

Point-of-care ultrasound for the detection of SCFE is a highly sensitive, non-ionizing imaging modality. Emergency physicians may choose to consider bedside ultrasound examination of the hip as an adjunct to plain radiography when evaluating a child with a limp.

Conflict of interest None. 


\section{References}

1. Rahme D, Comley A, Foster B et al (2006) Consequences of diagnostic delays in slipped capital femoral epiphysis. J Pediatr Orthop B 15(2):93-97

2. Green DW, Mogekwu N, Scher DM (2009) A modification of Klein's Line to improve sensitivity of the anterior-posterior radiography in slipped capital femoral epiphysis. J Pediatr Orthop 29(5):449-453

3. Terjesen T (1992) Ultrasonography for diagnosis of slipped capital femoral epiphysis. Comparison with radiography in 9 cases. Acta Orthop Scand 63(6):653-657
4. Magnano GM, Lucigrai G, De Filippi C et al (1998) Diagnostic imaging of the early slipped capital femoral epiphysis. Radiol Med 95(1-2):16-20

5. Kallio PE, Paterson DC, Foster BK et al (1993) Classification in slipped capital femoral epiphysis. Sonographic assessment of stability and remodeling. Clin Orthop Relat Res 294:196-203

6. Kallio PE, Lequesne GW, Paterson DC et al (1991) Ultrasonography in slipped capital femoral epiphysis. Diagnosis and assessment of severity. J Bone Joint Surg Br 73(6):884-889 